\title{
THE POLITICAL-MIIITARY RIVALRY FOR OPERATIONAL CONTROL IN U.S. MILITARY ACTIONS: A SOLDIER'S PERSPECTIVE
}

Colonel Lloyd J. Matthews, USA Retired Y

June 22, 1998 